\title{
Bioinformatic and mouse model reveal the potential high vulnerability of Leydig cells on SARS-CoV-2
}

\author{
Jiawei Zhang' ${ }^{1 \#}$, Yuqi Wu ${ }^{1 \#}$, Shulin $\mathrm{Li}^{1}$, Xiaobin Wang ${ }^{1}$, Rui Wang ${ }^{1}$, Xiangwei Wang ${ }^{1,2,3}$ \\ ${ }^{1}$ Department of Urology and Carson International Cancer Center, Shenzhen University General Hospital and Shenzhen University Clinical Medical \\ Academy Center, Shenzhen University, Shenzhen, China; ${ }^{2}$ Department of Urology, Southern University of Science and Technology Hospital, \\ Shenzhen, China; ${ }^{3}$ Department of Perioperative Medicine, Southern University of Science and Technology, Shenzhen, China \\ Contributions: (I) Conception and design: X Wang, R Wang; (II) Administrative support: R Wang, X Wang; (III) Provision of study materials or \\ patients: S Li; (IV) Collection and assembly of data: S Li; (V) Data analysis and interpretation: J Zhang, Y Wu; (VI) Manuscript writing: All authors; \\ (VII) Final approval of manuscript: All authors. \\ "These authors contributed equally to this work. \\ Correspondence to: Xiangwei Wang. Department of Urology, Southern University of Science and Technology Hospital, 6019 Liuxian Street, Xili \\ Avenue, Nanshan District, Shenzhen 518000, China. Email: winn0324@szu.edu.cn; Rui Wang. Department of Urology and Carson International \\ Cancer Center, Shenzhen University General Hospital and Shenzhen University Clinical Medical Academy Center, Shenzhen University, Shenzhen, \\ China. Email: 18664563718@163.com.
}

\begin{abstract}
Background: The severe acute respiratory syndrome coronavirus 2 (SARS-CoV-2) was first reported in China and spread rapidly since the end of 2019. Previous studies have confirmed that SARS-CoV-2 infects host cells via binding to angiotensin converting enzyme II (ACE2).

Methods: To explore the expression of ACE2 and the potential risk of infection in testis, we performed a bioinformatic analysis based on public databases, and conducted a pilot study using a mouse model. We also collected clinical follow-up date on male patients who had recovered from COVID-19 for 6 months.

Results: The results showed that the RNA expression of ACE2 was higher in testis compared with other organs. Single-cell analysis and immunocytochemistry further indicated that Leydig cells were at risk of SARS-CoV-2 infection. Green fluorescence was only detected in the Leydig cells after intratesticular injection of pseudovirus SARS-CoV-2 in the mouse model. In the clinical follow-up, serum total testosterone level was statistically lower in patients who had recovered from COVID-19 compared with healthy men $(\mathrm{P}=0.010)$.

Conclusions: The findings of the present study indicate the potential vulnerability of Leydig cells. It is important to monitor the reproductive system and its complications in male COVID-19 patients. Further studies are still needed on SARS-CoV-2-associated reproductive complications.
\end{abstract}

Keywords: Severe acute respiratory syndrome coronavirus 2 (SARS-CoV-2); testis; reproductive system; bioinformatic

Submitted Jan 27, 2021. Accepted for publication Apr 15, 2021.

doi: $10.21037 /$ atm-21-936

View this article at: http://dx.doi.org/10.21037/atm-21-936

\section{Introduction}

The severe acute respiratory syndrome coronavirus 2 (SARS$\mathrm{CoV}-2$ ) was first reported in China and spread rapidly since the end of 2019. SARS-Cov-2 belongs to the same subgenus as the SARS virus and the Middle East respiratory syndrome virus (MERS), as determined by full-genome sequencing analysis method (1). SARS-CoV-2 also has the closest RNA sequence compared with the coronavirus from the Rhinolophus bat (2), which indicates that this was possibly the primary source or an intermediate host of coronavirus 2019 (COVID-19). The main clinical manifestations of COVID-19 are fever, cough, dyspnea, and bilateral lung infiltration on chest imaging. Although most currently 
reported cases are not severe, about $20 \%$ of patients diagnosed are critically ill with respiratory failure, septic shock, or other organ failure requiring intensive care (3-5).

Based on previous studies, both SARS and SARS$\mathrm{CoV}-2$ have a similar spike in glycoprotein (S protein), which can strongly bind to cell receptor, angiotensin converting enzyme II (ACE2), and induce infection to human cells $(1,2)$. The multiplication of SARS-CoV-2 will cause immune response after entering the host (6). The confirmed transmission routes of SARS-CoV-2 are via respiratory droplets and direct contact as SARS because of the high expression of ACE2 on type II alveolar cells of lung (7). Therefore, investigating the expression of ACE2 in human cells is considered to be related to the susceptibility of SARS-CoV-2. Previous studies have confirmed the relationship between the differential expression of ACE2 in human systems and clinical symptoms reported for each system, such as dyspnea in the respiratory system, diarrhea in the digestive system, and acute kidney injury in the urinary system $(2,8)$.

In their study, La Marca et al. reported on a COVID-19 case in April presenting with heavy testicular pain as the first symptom (9). At the same time, Gagliardi et al. reported on a young boy who diagnosed with orchiepididymitis and COVID-19 (10). Some viruses, such as the mumps virus, HIV, and Zika virus, may affect the testis (11); however, it is unclear whether SARS-CoV-2 can directly invade or indirectly damage the male reproductive system. In the present study, we explored the expression of ACE2 in various organs and investigated the detail existence of ACE2 in the testis by single-cell transcriptomes based on public databases. We further investigated the vulnerability of testis on SARS-Cov-2 in the animal model and conducted a clinical follow-up. We hypothesized that SARS-CoV-2 may infect Leydig cells, but not semen in testis, therefore leading to orchitis. These findings emphasize the importance of monitoring complications in the male reproductive system, and could contribute to the management of COVID-19 patients. We present the following article in accordance with the ARRIVE reporting checklist (available at http:// dx.doi.org/10.21037/atm-21-936).

\section{Methods}

\section{Public data sources}

The bodymap of the median expression of ACE2 was obtained from the Gene Expression Profiling Interactive
Analysis (http://gepia2.cancer-pku.cn/\#general). The expression profiles of ACE2 in adult normal tissue, including the testis, kidney, fallopian tube, kidney cortex, seminal vesicle, vagina, ovary, bladder, lung, prostate, placenta, tonsil, epididymis, vas deferens, renal pelvis, endometrium, uterus, and endocervix, were obtained from the Expression Atlas. The single-cell data of testis were from GSE124263. Images of immunohistochemistry sections of human testis with ACE2 staining (patient ID: 2257) was acquired from The Human Protein Atlas.

\section{Quality control}

Low-quality cells with expressed genes $(<1,000$ or $>20,000)$ were removed. We further required the percentage of Unique Molecular Identifiers (UMIs) mapped to mitochondrial or ribosomal genes to be $<10 \%$.

\section{Dimension reduction, cell clustering, gene expression analysis, and cell type identification}

The single-cell data expression matrix was processed with the R package "Seurat" (version 3.1). First, we utilized "LogNormalize" to normalize the single-cell gene expression data. We then identified the highly variable genes using the function "FindVariableGenes" by "vst" method. The principle component analysis on the singlecell expression matrix with significant highly variable genes was performed using the "RunPCA" function. We then used the t-Distributed Stochastic Neighbor Embedding (TSNE) function to obtain cell scatterplots. Gene expression markers of identity classes were acquired by the "FindMarkers" function (min.pct $=0.25$, logfc.threshold $=0.25$ ). Feature plots and violin plots were generated using "Seurat" to show imputed gene expression (12-15). Cell type identification was carried out using canonical marker genes (16).

\section{Animals and pseudovirus SARS-CoV-2}

Fifteen adult male mice (C57BL/6N, 8 weeks old, mean weight $22.6 \mathrm{~g}$ ) were obtained from Beijing Vital River Laboratory Animal Technology (Beijing, China), and maintained under standardized conditions with a 12-h lightdark cycle and feed. Animal experiments were approved by the Medical Animal Care \& Welfare Committee of Shenzhen University Medical School in compliance with guidelines for the care and use of animals. Twelve mice were 
randomized into the test group and control group at a ratio of 1:1. The pseudovirus SARS-CoV-2 was purchased from ACE biolabs $\left(2 \times 10^{7} \mathrm{U} / \mathrm{mL}\right.$; Suzhou China). The pseudovirus SARS-CoV-2 contains the SARS-CoV-2 S protein as a surface capsid glycoprotein and Green Fluorescent Protein (GFP) as a pseudovirus model, which make it a replicationdefective virus that can only infect the target cells, but does not generate new virus particles. Fluorescence microscopy was used to determine the efficiency and activity of its infection.

\section{Immunobistochemistry}

Three adult male mice in the control group were killed to investigate the distribution of ACE2 in mouse testicular tissue. Testis was perfusion fixed with a testicular tissue fixative (Servicebio, Wuhan, China) and processed into paraffin. The slides were placed in $3 \%$ hydrogen peroxide solution and incubated at room temperature under dark conditions for $25 \mathrm{~min}$ to block endogenous peroxidase. In total, 3\% bovine serum albumin (Gibco, Grand Island, NY, USA) was used to block non-specific binding sites for $30 \mathrm{~min} ; 2 \mu \mathrm{g} / \mathrm{mL}$ ACE2 antibody (Abcam, Cambridge, MA, USA) was subsequently added to the sections, and the sections were incubated overnight at $4{ }^{\circ} \mathrm{C}$. Secondary goat anti-rabbit antibody (1:3,000; Abcam, Cambridge, MA, USA) was added for $50 \mathrm{~min}$. 3,3'-Diaminobenzidinetetrachloride color-developing solution (1:100; Dako, Glostrup, Denmark) was added and stopped when the positive color was brownish-yellow. The nuclei were restained with hematoxylin and then dehydrated and sealed. Sections were detected by microscope (Eclipse TI-SR; Nikon) and photographed using Nikon DS-U3.

\section{Intratesticular injection and detection of infection}

Twelve mice were anesthetized with $4 \%$ chloral hydrate ( $3 \mathrm{~mL} / \mathrm{kg}$ ) by intraperitoneal injection. In total, $100 \mu \mathrm{L}$ pseudovirus SARS-CoV-2 $\left(2 \times 10^{7} \mathrm{U} / \mathrm{mL}\right)$ was injected slowly into the interstitial space of each testis using a 30-gauge needle after anesthesia. Normal saline for intratesticular injection was used for the control group, as previously described. All mice were killed 1 week later and the testis were collected for paraffin embedding. The nuclei were stained by 4',6'-diamidino-2-phenylindole dihydrochloride (DAPI, no dilution; Servicebio, Wuhan, China). GFP was detected by fluorescence microscopy (Eclipse TI-SR; Nikon) and photographed using Nikon DS-U3.

\section{Laboratory test for serum sex hormones}

All procedures performed in this study involving human participants were in accordance with the Declaration of Helsinki (as revised in 2013). The ethics committee of Shenzhen University General Hospital approved the present study. Patient consents were obtained for the human research. We obtained serum from male patients who had recovered from COVID-19 for 6 months and age-matched controls. Serum sex hormones, including testosterone (Cusabio, Wuhan, China), follicle stimulating hormone (FSH; Cusabio, Wuhan, China), luteinizing hormone (LH; Cusabio, Wuhan, China) levels, were assayed by enzymelinked immunosorbent assay method.

\section{Statistical analysis}

SPSS (version 25.0; IBM, Armonk, NY, USA) was used for the statistical analyses. $\mathrm{P}<0.05$ was considered statistically significant. Descriptive statistics for continuous variables were expressed as mean \pm standard deviation. The $t$-test was used to compare age, body mass index (BMI), testosterone, and LH among the groups; Mann-Whitney U-test was used to compare FSH as the heteroscedasticity.

\section{Results}

\section{Expression of ACE2 in different tissues based on $R N A$ sequencing}

The bodymap of the median expression of ACE2 in normal samples revealed that ACE2 was widely expressed in multiple organs, including the lung, esophagus, colon, uterus, ovary, prostate, kidney ectocervix, and testis (Figure $1 A$ ). The data were derived from the RNA sequencing datasets of The Cancer Genome Atlas Program and the GenotypeTissue Expression databases, and were then displayed using $\log 2$ [transcripts per million (TMP) +1] after homogenization. In addition to the reported respiratory system (lung log2TMP+1: 0.89), digestive system (esophagus $\log 2 \mathrm{TMP}+1: 1.43$, stomach $\log 2 \mathrm{TMP}+1: 0.4$, colon $\log 2 \mathrm{TMP}+1: 1.04$ ), and urinary system (kidney $\log 2 \mathrm{TMP}+1$ : 3.73, bladder $\log 2 \mathrm{TMP}+1: 0.67)$, ACE2 was also expressed in male and female reproductive systems (prostate log2TMP+1: 0.5 , ovary $\log 2 \mathrm{TMP}+1: 0.94$, ectocervix $\log 2 \mathrm{TMP}+1: 0.41$, and testis $\log 2 \mathrm{TMP}+1: 5.16)$. According to the heatmap we obtained from the Expression Atlas, it was interesting to see that the expression of ACE2 was significantly higher in testis than any other organ (Figure 1B). 

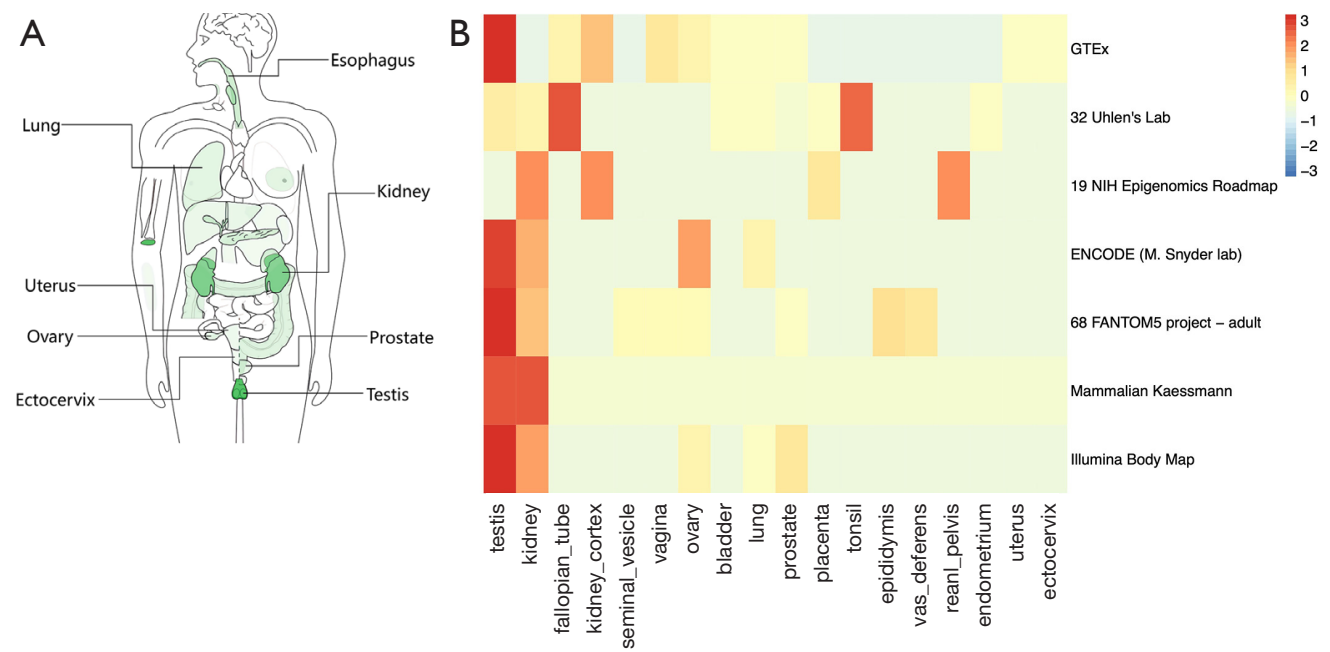

Figure 1 Angiotensin converting enzyme II (ACE2) expression analysis from RNA sequencing databases. (A) Bodymap of ACE2 median expression, measured as $\log 2$ (transcripts per million +1 ) in different organs, was obtained from Gene Expression Profiling Interactive Analysis. (B) Heatmap of differentially expressed ACE2 in the respiratory system, urinary system, and reproductive system organs were acquired from the Expression Atlas, including Genotype-Tissue Expression, 32 Uhlen's Lab, 19 NIH Epigenomics Roadmap, ENCODE, 68 FANTOM5 project-adult, Mammalian Kaessmann, and Illumina Body Map databases.

\section{Cell type-specific ACE2 expression in buman testis}

To further explore the expression of ACE2 in the testis, we analyzed the single cell RNA (scRNA) sequencing dataset of human testis. Eight clusters were identified via $\mathrm{t}$-distributed stochastic neighbor embedding ( $\mathrm{tSNE}$ ) based on known cell-type marker expression (Figure S1 and Figure 2A). Detailed representative markers of Leydig cells, macrophages, endothelial cells, myoid cells, sperm, spermatogonial stem cells, primary spermatocytes are shown in Figures S2-S8, respectively. The tSNE feature plot and violin plot indicated that ACE2 was mainly expressed in clusters 0-2, which represented Leydig cells, myoid cells, and spermatogonial stem cells, respectively (Figure 2B,C).

\section{Expression of ACE2 in testis based on immunocytochemistry}

Although the expression level of mRNA could reflect the protein content to a certain extent, the expression level of RNA is often not directly proportional to the expression level of protein, due to different regulatory processes in the process of mRNA translation in different tissues (10). Therefore, we obtained ACE2 immunohistochemical profiles of testis from the public database of The
Human Protein Atlas. Interestingly, we found that ACE2 was significantly highly stained in Leydig cells and spermatogonia in human testis tissue (Figure $3 A$ ). Furthermore, we also found a similar expression of ACE2 in mouse testis, and the staining level in Leydig cells was higher than that in spermatogonia (Figure 3B).

\section{Immunofluorescence localization of pseudovirus SARS- CoV-2 after intratesticular injection}

Blue staining of nuclei could be observed by fluorescence microscope in all testicular tissues. For control group, no green fluorescence was detected in the testicular tissue (Figure $4 A$ ). In the pseudovirus SARS-CoV-2 intratesticular injection group, GFP was detected in the Leydig cells (Figure 4B). No green fluorescence was observed in spermatogonia, sperm, macrophages, and other cells, except Leydig cells.

\section{Clinical follow-up of serum sex hormones}

Twenty male patients with laboratory-confirmed SARSCoV-2, who had recovered from COVID-19 for over 6 months, were included in the present study. The same number of age-matched controls were also included. No testicular symptoms were reported before and during the 

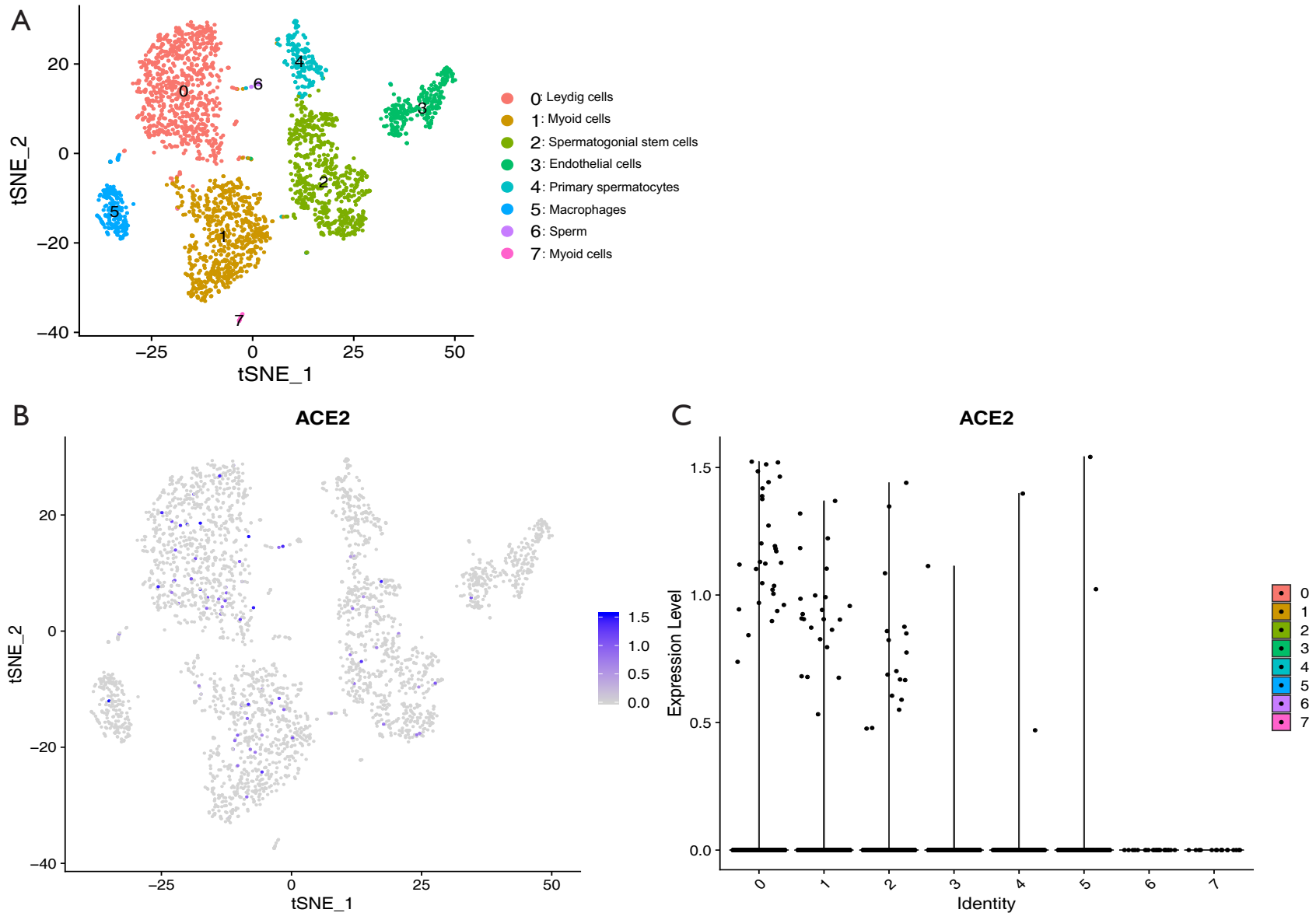

Figure 2 Testicular cell single cell RNA sequencing data analysis. (A) Cells were categorized into 8 clusters. (B) The t-distributed stochastic neighbor embedding feature plot showed that the cluster of cells with ACE2 expression. (C) Violin plot showing angiotensin converting enzyme II expression distribution of different cell clusters.
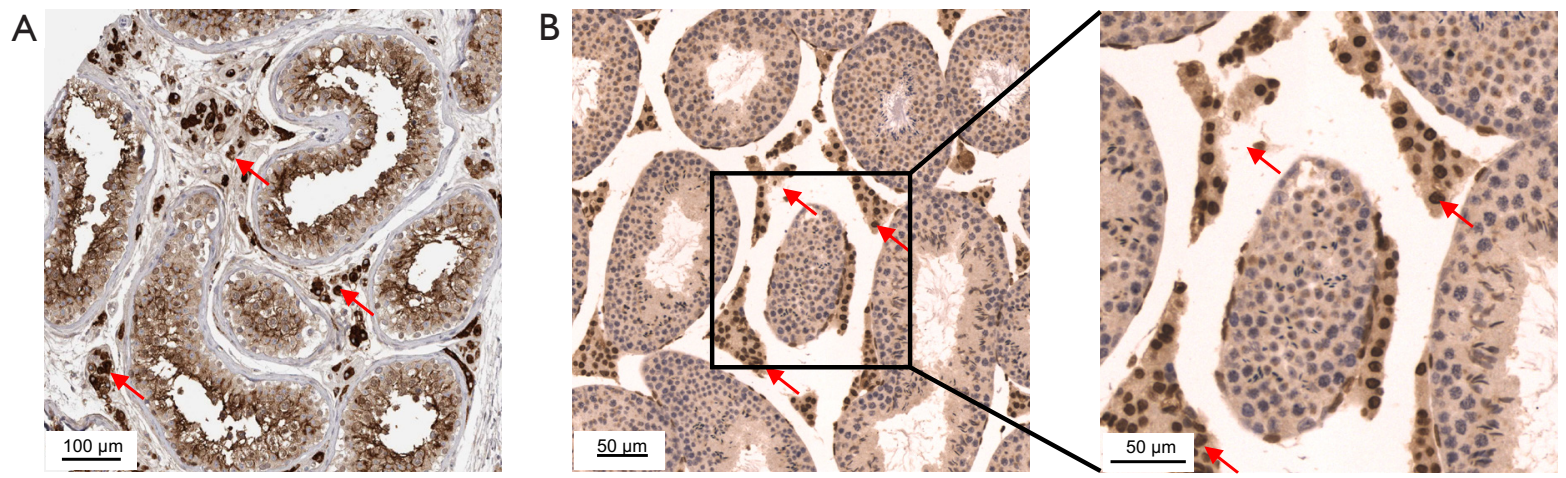

Figure 3 ACE2 distribution in human and mouse testicular tissue. (A) Immunohistochemical profiles were obtained from The Human Protein Atlas. Human testicular tissue with angiotensin converting enzyme II (ACE2) stain was taken from a 26-year-old male (patient ID: 2257). (B) Immunohistochemical distribution of ACE2 in mouse testicular tissue $(20 \times$, 40×). Arrow shows Leydig cells with high ACE2 expression. 

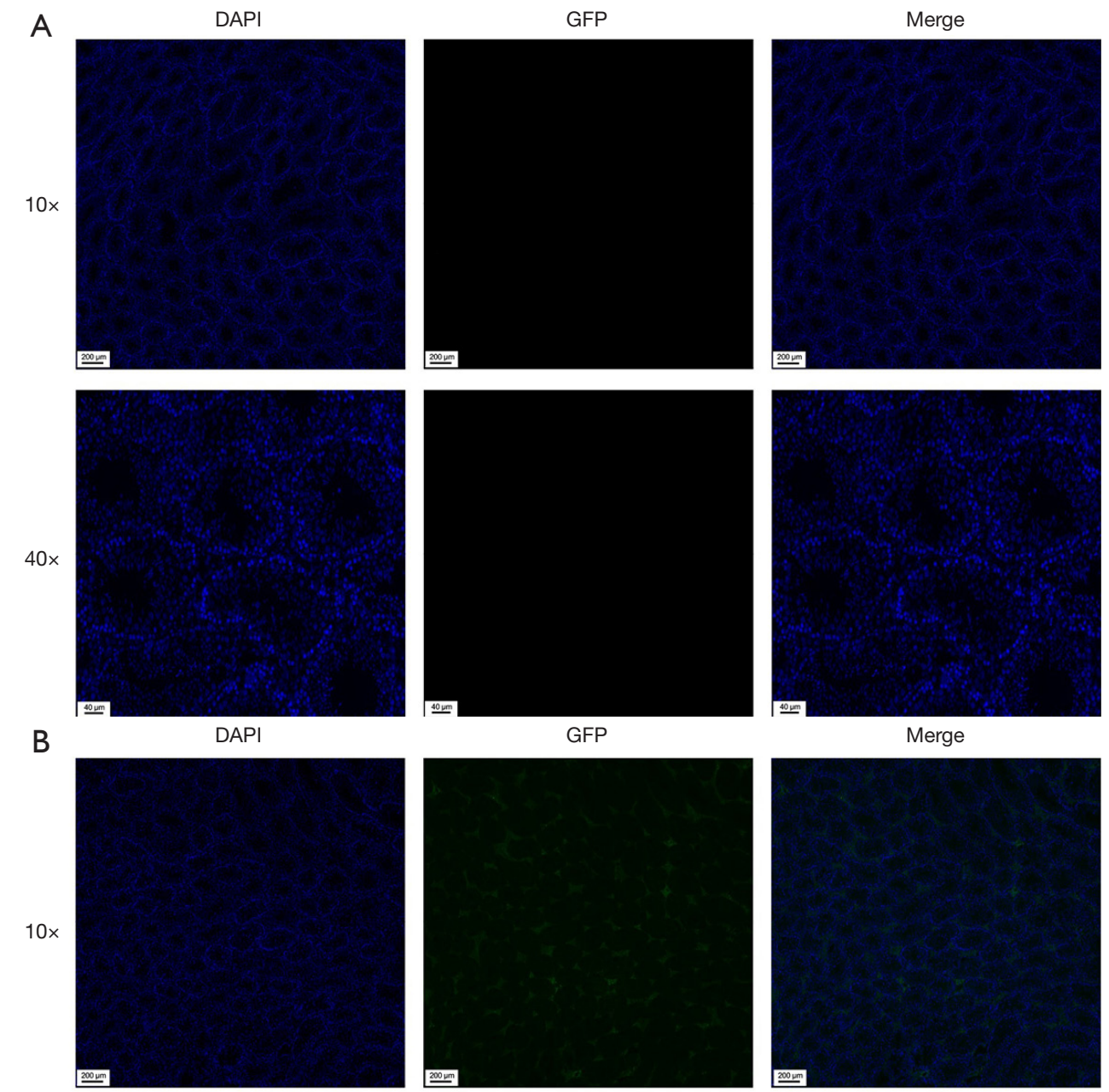

GFP

Merge
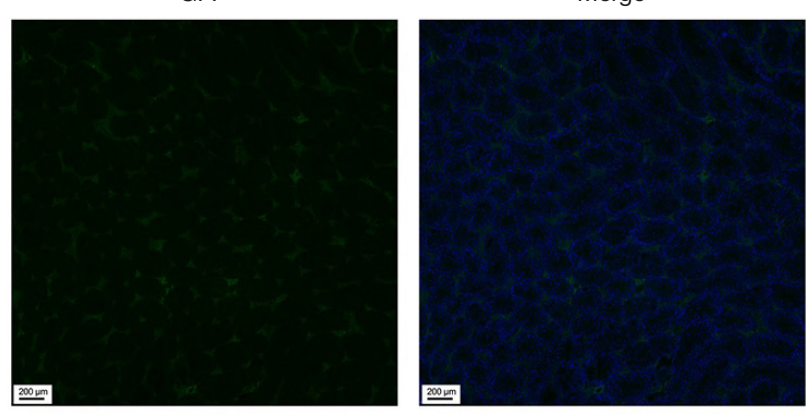

$40 x$
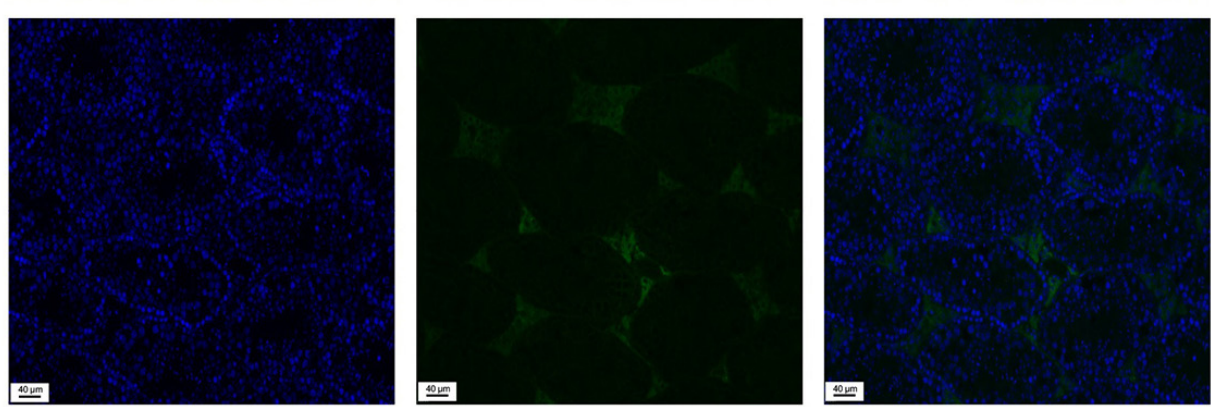

Figure 4 Immunofluorescence detected the location of pseudovirus Severe acute respiratory syndrome coronavirus 2 (SARS-CoV-2) after intratesticular injection. (A) No green fluorescence was observed in the negative control. (B) Immunofluorescence localization of green fluorescent protein (GFP) (green) and 4',6'-diamidino-2-phenylindole dihydrochloride (DAPI) (blue) is shown. Intratesticular injection of pseudovirus SARS-CoV-2 resulted in the selective transduction of Leydig cells in mice. 
Table 1 Serum sexual related hormone levels on coronavirus 2019 (COVID-19) patients and controls

\begin{tabular}{lccc}
\hline Variable & $\begin{array}{c}\text { Men with COVID-19 } \\
(\mathrm{n}=20)\end{array}$ & $\begin{array}{c}\text { Age-matched } \\
\text { controls }(\mathrm{n}=20)\end{array}$ & P value \\
\hline Age (years) & $29.15 \pm 9.71$ & $29.45 \pm 7.65$ & 0.914 \\
BMI & $23.60 \pm 3.44$ & $25.22 \pm 3.60$ & 0.154 \\
$\begin{array}{l}\text { Testosterone } \\
\text { (nmol/L) }\end{array}$ & $14.94 \pm 4.02$ & $18.76 \pm 4.89$ & $0.010^{\star}$ \\
FSH (IU/L) & $4.06 \pm 1.23$ & $4.99 \pm 2.15$ & 0.221 \\
LH (IU/L) & $4.49 \pm 1.12$ & $5.10 \pm 1.92$ & 0.265 \\
\hline
\end{tabular}

*, statistically significant at $\mathrm{P}<0.05$. Data are presented as mean \pm standard deviation. BMI, body mass index; $\mathrm{FSH}$, follicle stimulating hormone; LH, luteinizing hormone.

follow-up. The mean age of patients who had recovered from COVID-19 was $29.15 \pm 9.71$ years, while the mean age of controls was $29.45 \pm 7.65(\mathrm{P}=0.914)$. There were no statistical differences in $\mathrm{BMI}$ between the groups $(\mathrm{P}=0.154)$. Serum total testosterone level was lower in COVID-19 patients $(14.94 \pm 4.02)$ compared with controls $(\mathrm{P}=0.010)$. However, there were no significant differences in terms of testosterone level, which remained within the normal range. No significant differences were observed in terms of the average levels of FSH and LH between COVID-19 patients and controls ( $\mathrm{P}=0.221$ and $\mathrm{P}=0.265$, respectively) (Table 1 ).

\section{Discussion}

The findings of the present study indicate a higher expression of ACE2 in testis compared with the lung by providing the mRNA and protein expression of the receptor ACE2 via interrogating public databases. SARS-CoV-2 may infect the testis, as it has potential high vulnerability. We further investigated the vulnerable cell type of testis on the single-cell level based on the Gene Expression Omnibus (GEO) database and verified our hypothesis that Leydig cells are possible target of SARS-CoV-2 through the intratesticular injection of a pseudovirus in mice. Leydig cells are endocrine cells in the testicular stroma of mammals that synthesize and secrete androgen. Leydig cell infection may cause inflammatory changes in the testis and decrease androgen synthesis, which may indirectly lead to sperm production disorders and even infertility. Zhao et al. reported that SARS was detected in testicular epithelial cells and Leydig cells in two autopsy cases who died of SARS (17). Our results are consistent with those of Yang et al., who reported significant seminiferous tubular injury, reduced Leydig cells, and mild lymphocytic inflammation in COVID-19 patients (18). Therefore, we speculated that SARS-CoV-2 may also exist in the testis, especially in Leydig cells, which may cause orchitis and orchiepididymitis. Orchitis or orchiepididymitis should be considered as one of the complications of COVID-19 during treatment and follow-up. Another concern is whether the SARS-CoV-2 can infect the spermatogonia or sperm directly. Li et al. reported on 6 SARS-CoV-2 patients, including 2 who had recovered (19). However, most studies have indicated that SARS-CoV-2 was not detected in semen (20-22). In a cohort study, Holtmann et al. found an impairment in sperm quality due to moderate COVID-19 infection, although no SARS-CoV-2 RNA was detected in the samples (23). In our study, neither the expression of ACE2 on sperm nor pseudovirus transfection into spermatogonia in vivo was found. This indicates that semen is unlikely to be infected compared with Leydig cells, although both highly express ACE2. In addition, some reports have demonstrated that a lower level of testosterone in COVID-19 patients can result in poor prognosis and mortality, which is consistent with our follow-up results on sexual sex hormones $(24,25)$. However, the average level of testosterone followed in our study continued to be normal after 6 months, which suggests that inflammatory damage caused by intratesticular infection may be low or reversible.

The present study has some limitation. Although we constructed a single-cell atlas on the testis, the number of total cells included in our analysis was low, which may lead to bias in analyzing the expression of ACE2. The reasons for this may be the strict criteria in quality control and the limited datasets uploaded in the GEO database. However, the 2 nd-generation sequencing results and immunohistochemistry of ACE2 showed in our study could be considered additional evidence. We were unable to use human ACE2 transgenic mice for research in the in vivo experiment due to limited conditions, which may better mimic the process of human testicular infection. Also, the binding ability of the pseudovirus may not be as good as SARS-CoV-2, and may not simulate the subsequent damage on testicular tissue. In addition, we followed-up patients who had recovered from SARS-CoV-2 infection after 6 months; a longer follow-up is needed to rule out longterm complications, such as oligospermia and infertility.

In conclusion, the findings of the present study indicate the potential vulnerability of SARS-CoV-2 on testis, especially in Leydig cells. Orchitis and orchiepididymitis 
may be the post-infection clinical manifestation, but there is no evidence to support the possibility of sperm infection or infertility. Further studies are still needed on SARS-CoV-2associated reproductive complications.

\section{Acknowledgments}

Funding: This work was supported by the fellowship of China Postdoctoral Science Foundation (No. 2020M670048ZX) and the Science and Technology Innovation Commission Foundation of Shenzhen (Grant No. JCYJ20190808141013454 and JCYJ20180305124827261).

\section{Footnote}

Reporting Checklist: The authors have completed the ARRIVE reporting checklist. Available at http://dx.doi. org/10.21037/atm-21-936

Data Sharing Statement: Available at http://dx.doi. org/10.21037/atm-21-936

Conflicts of Interest: All authors have completed the ICMJE uniform disclosure form (available at http://dx.doi. org/10.21037/atm-21-936). The authors have no conflicts of interest to declare.

Ethical Statement: The authors are accountable for all aspects of the work in ensuring that questions related to the accuracy or integrity of any part of the work are appropriately investigated and resolved. Animal experiments were approved by the Medical Animal Care \& Welfare Committee of Shenzhen University Medical School in compliance with guidelines for the care and use of animals. All procedures performed in this study involving human participants were in accordance with the Declaration of Helsinki (as revised in 2013). All research procedures were approved by the Medical Ethical Committee of Shenzhen University Medical School. Patient consents were obtained for the human research.

Open Access Statement: This is an Open Access article distributed in accordance with the Creative Commons Attribution-NonCommercial-NoDerivs 4.0 International License (CC BY-NC-ND 4.0), which permits the noncommercial replication and distribution of the article with the strict proviso that no changes or edits are made and the original work is properly cited (including links to both the formal publication through the relevant DOI and the license). See: https://creativecommons.org/licenses/by-nc-nd/4.0/.

\section{References}

1. Zhou P, Yang XL, Wang XG, et al. A pneumonia outbreak associated with a new coronavirus of probable bat origin. Nature 2020;579:270-3.

2. Lu R, Zhao X, Li J, et al. Genomic characterisation and epidemiology of 2019 novel coronavirus: implications for virus origins and receptor binding. Lancet 2020;395:565-74.

3. Huang C, Wang Y, Li X, et al. Clinical features of patients infected with 2019 novel coronavirus in Wuhan, China. Lancet 2020;395:497-506.

4. Guan WJ, Ni ZY, Hu Y, et al. Clinical Characteristics of Coronavirus Disease 2019 in China. N Engl J Med 2020;382:1708-20.

5. Chen N, Zhou M, Dong X, et al. Epidemiological and clinical characteristics of 99 cases of 2019 novel coronavirus pneumonia in Wuhan, China: a descriptive study. Lancet 2020;395:507-13.

6. Alipoor SD, Mortaz E, Jamaati H, et al. COVID-19: Molecular and Cellular Response. Front Cell Infect Microbiol 2021;11:563085.

7. Hatmal MM, Alshaer W, Al-Hatamleh MAI, et al. Comprehensive Structural and Molecular Comparison of Spike Proteins of SARS-CoV-2, SARS-CoV and MERS-CoV, and Their Interactions with ACE2. Cells 2020;9:2638.

8. Hamming I, Cooper ME, Haagmans BL, et al. The emerging role of ACE2 in physiology and disease. J Pathol 2007;212:1-11.

9. La Marca A, Busani S, Donno V, et al. Testicular pain as an unusual presentation of COVID-19: a brief review of SARS-CoV-2 and the testis. Reprod Biomed Online 2020;41:903-6.

10. Gagliardi L, Bertacca C, Centenari C, et al. Orchiepididymitis in a Boy With COVID-19. Pediatr Infect Dis J 2020;39:e200-e202.

11. Liu $W$, Han $\mathrm{R}, \mathrm{Wu} \mathrm{H}$, et al. Viral threat to male fertility. Andrologia 2018;50:e13140.

12. McDavid A, Finak G, Chattopadyay PK, et al. Data exploration, quality control and testing in single-cell qPCR-based gene expression experiments. Bioinformatics 2013;29:461-7.

13. Love MI, Huber W, Anders S. Moderated estimation of fold change and dispersion for RNA-seq data with 
DESeq2. Genome Biol 2014;15:550.

14. Trapnell C, Cacchiarelli D, Grimsby J, et al. The dynamics and regulators of cell fate decisions are revealed by pseudotemporal ordering of single cells. Nat Biotechnol 2014;32:381-6.

15. Finak G, McDavid A, Yajima M, et al. MAST: a flexible statistical framework for assessincg transcriptional changes and characterizing heterogeneity in single-cell RNA sequencing data. Genome Biol 2015;16:278.

16. Guo J, Grow EJ, Mlcochova H, et al. The adult human testis transcriptional cell atlas. Cell Res 2018;28:1141-57.

17. Zhao J, Zhou G, Sun Y. SARS coronavirus could cause multi-organ infection. Medical Journal of Chinese People's Liberation Army 2003;28:697-8.

18. Yang $M$, Chen $S$, Huang B, et al. Pathological Findings in the Testes of COVID-19 Patients: Clinical Implications. Eur Urol Focus 2020;6:1124-9.

19. Li D, Jin M, Bao P, et al. Clinical Characteristics and Results of Semen Tests Among Men With Coronavirus Disease 2019. JAMA Netw Open 2020;3:e208292.

20. Guo L, Zhao S, Li W, et al. Absence of SARS-CoV-2 in semen of a COVID-19 patient cohort. Andrology

Cite this article as: Zhang J, Wu Y, Li S, Wang X, Wang R, Wang X. Bioinformatic and mouse model reveal the potential high vulnerability of Leydig cells on SARS-CoV-2. Ann Transl Med 2021;9(8):678. doi: 10.21037/atm-21-936
2021;9:42-7.

21. Pan F, Xiao X, Guo J, et al. No evidence of severe acute respiratory syndrome-coronavirus 2 in semen of males recovering from coronavirus disease 2019. Fertil Steril 2020;113:1135-9.

22. Massarotti C, Garolla A, Maccarini E, et al. SARS-CoV-2 in the semen: Where does it come from? Andrology 2021;9:39-41.

23. Holtmann N, Edimiris P, Andree M, et al. Assessment of SARS-CoV-2 in human semen-a cohort study. Fertil Steril 2020;114:233-8.

24. Rastrelli G, Di Stasi V, Inglese F, et al. Low testosterone levels predict clinical adverse outcomes in SARS-CoV-2 pneumonia patients. Andrology 2021;9:88-98.

25. Çayan S, Uğuz M, Saylam B, et al. Effect of serum total testosterone and its relationship with other laboratory parameters on the prognosis of coronavirus disease 2019 (COVID-19) in SARS-CoV-2 infected male patients: a cohort study. Aging Male 2020;23:1493-503.

(English Language Editor: R. Scott) 

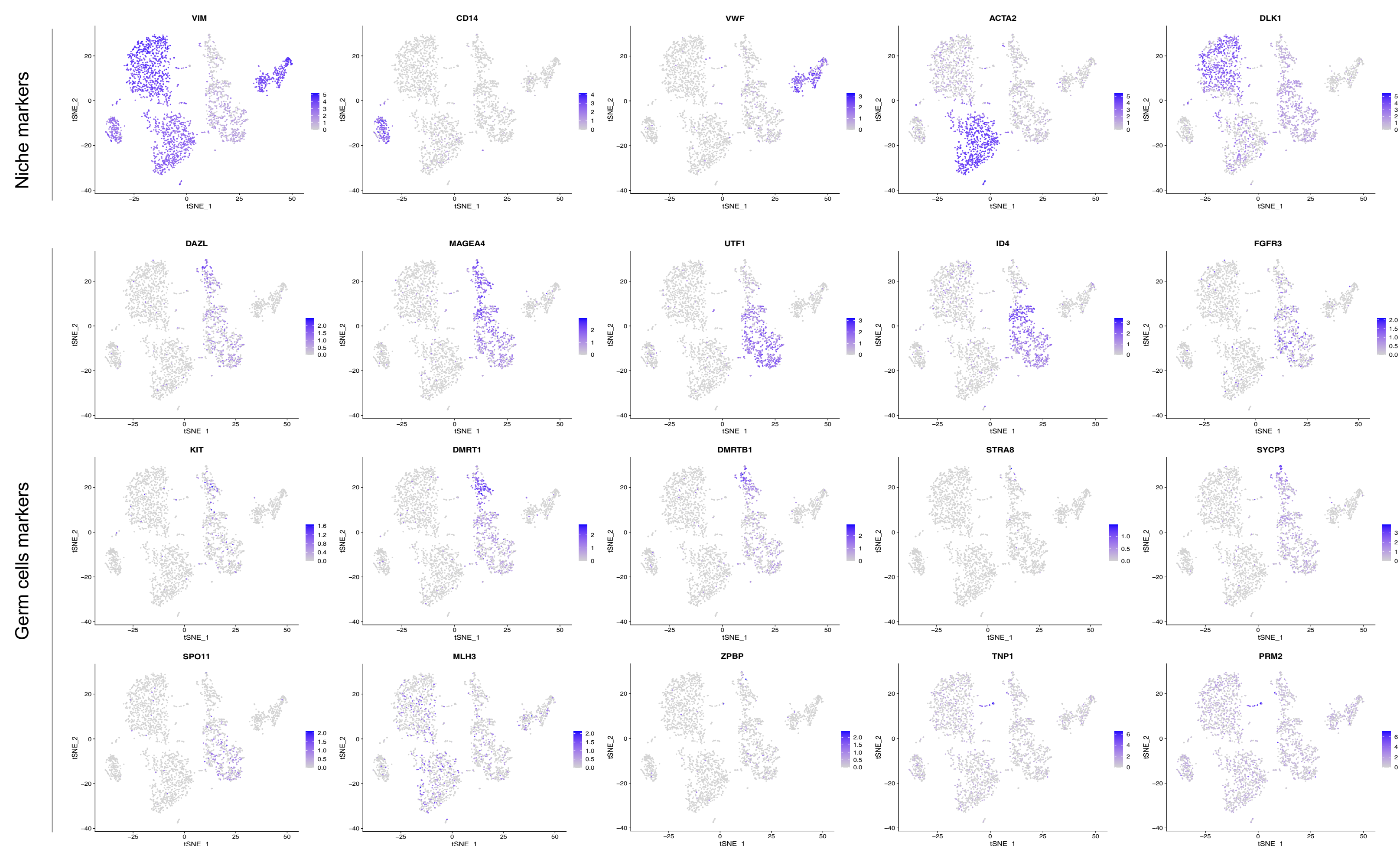

Figure S1 Expression of selected markers (somatic/niche cell markers and germ cell markers) are shown on the t-distributed stochastic neighbor embedding plot. Blue indicates high expression, and gray indicates low or no expression. 

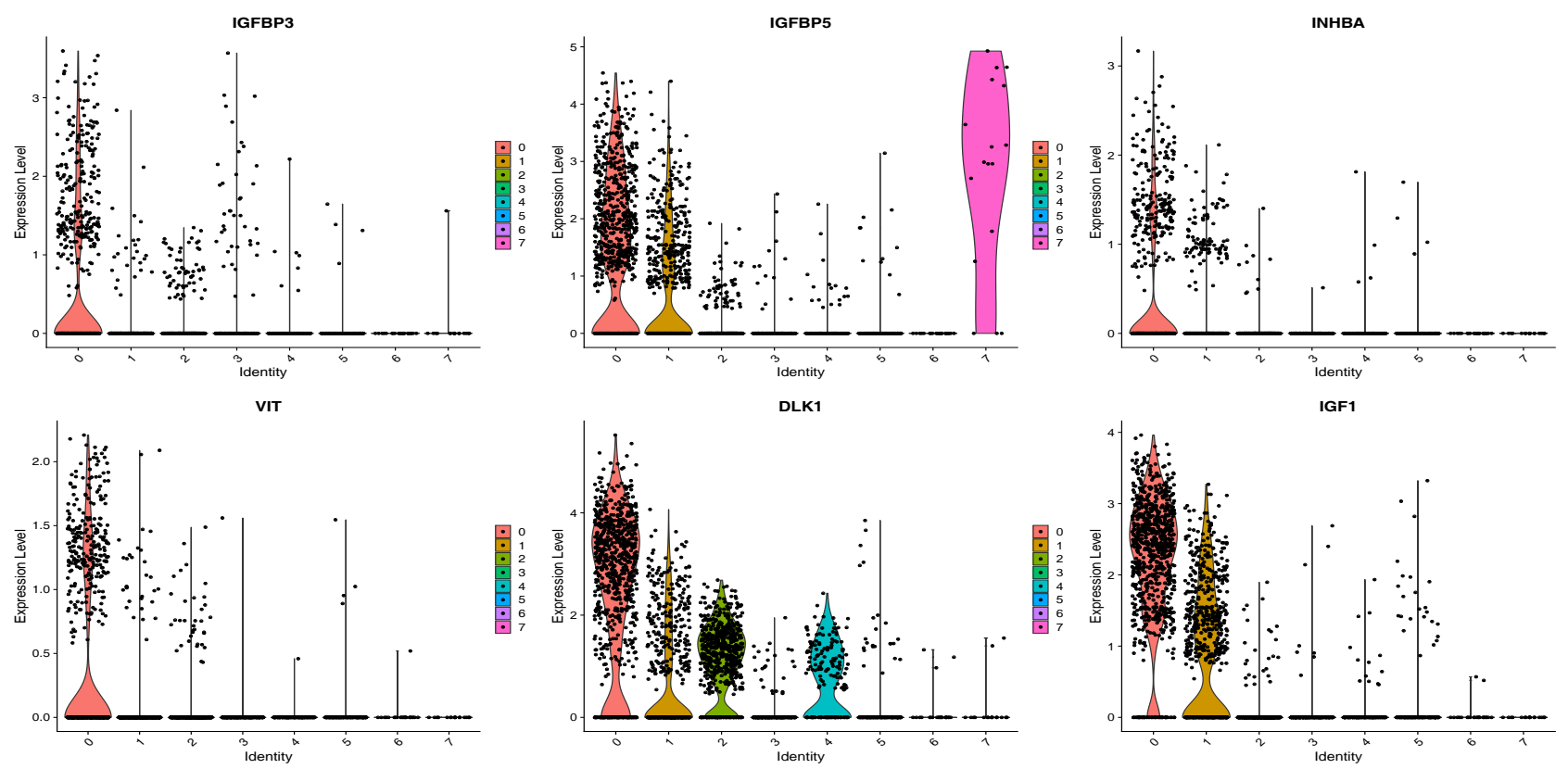

Figure S2 Gene expression patterns (violin plot) of selected markers in Leydig cells.
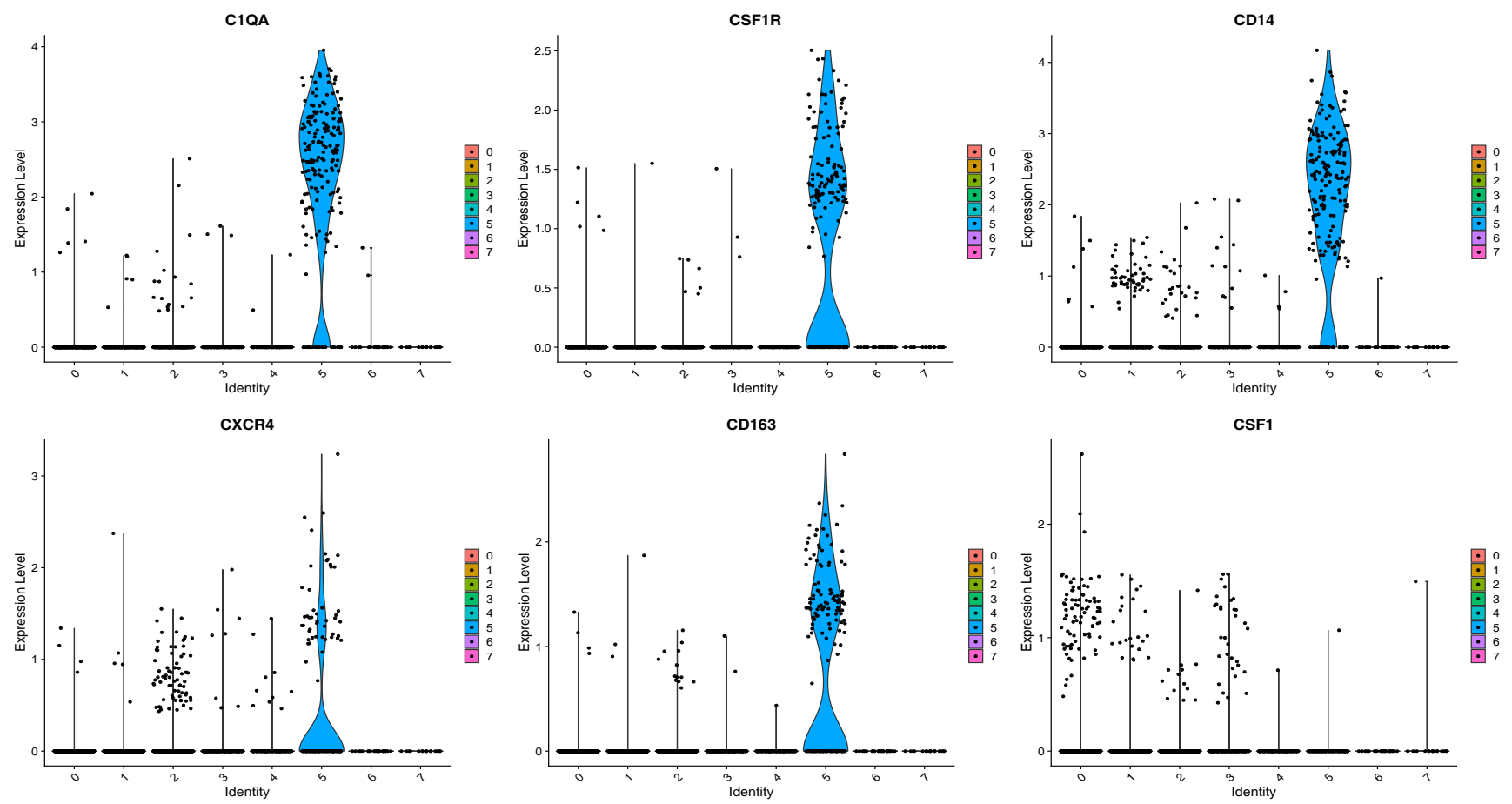

Figure S3 Gene expression patterns (violin plot) of selected markers in macrophages. 

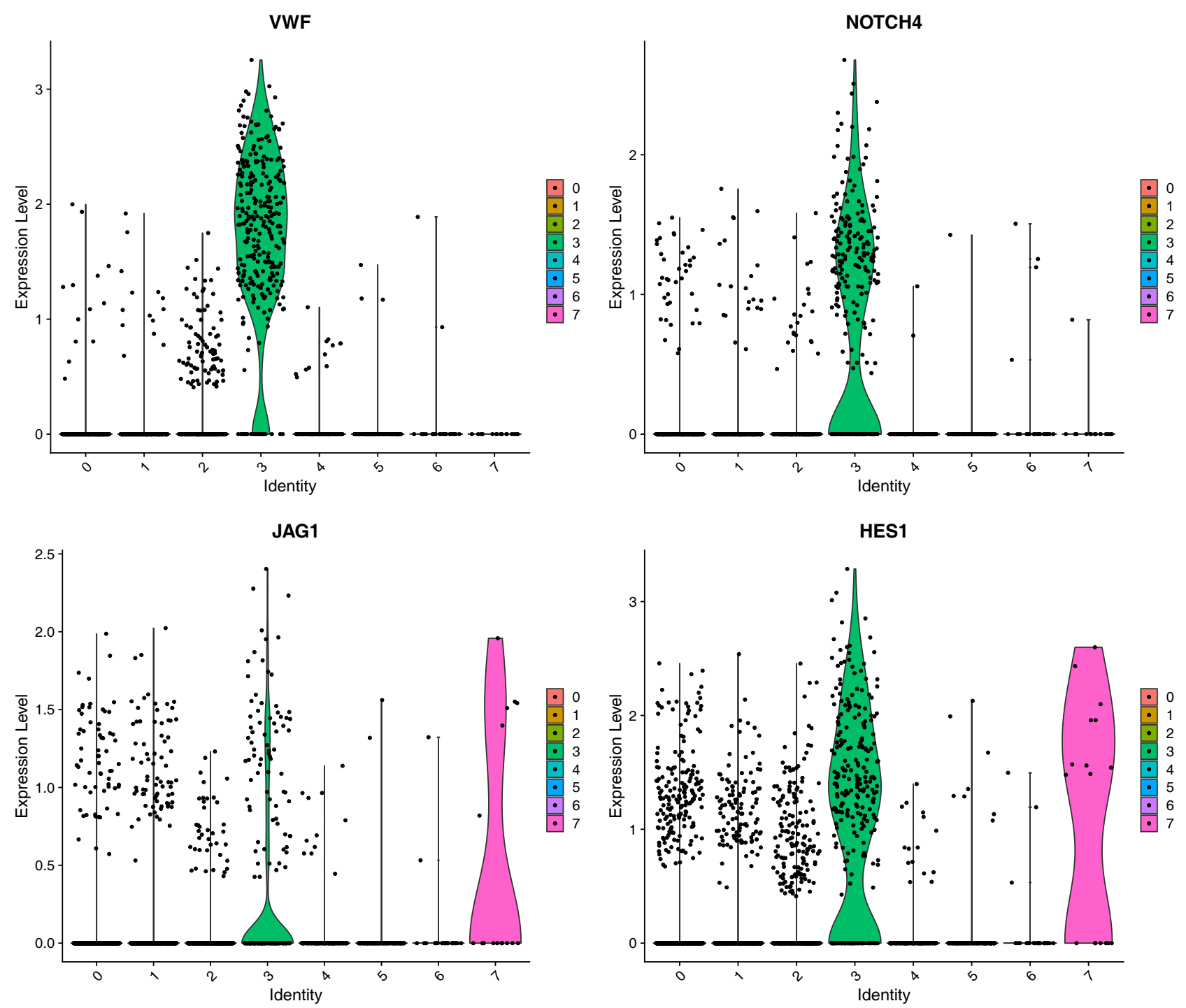

Figure S4 Gene expression patterns (violin plot) of selected markers in endothelial cells. 

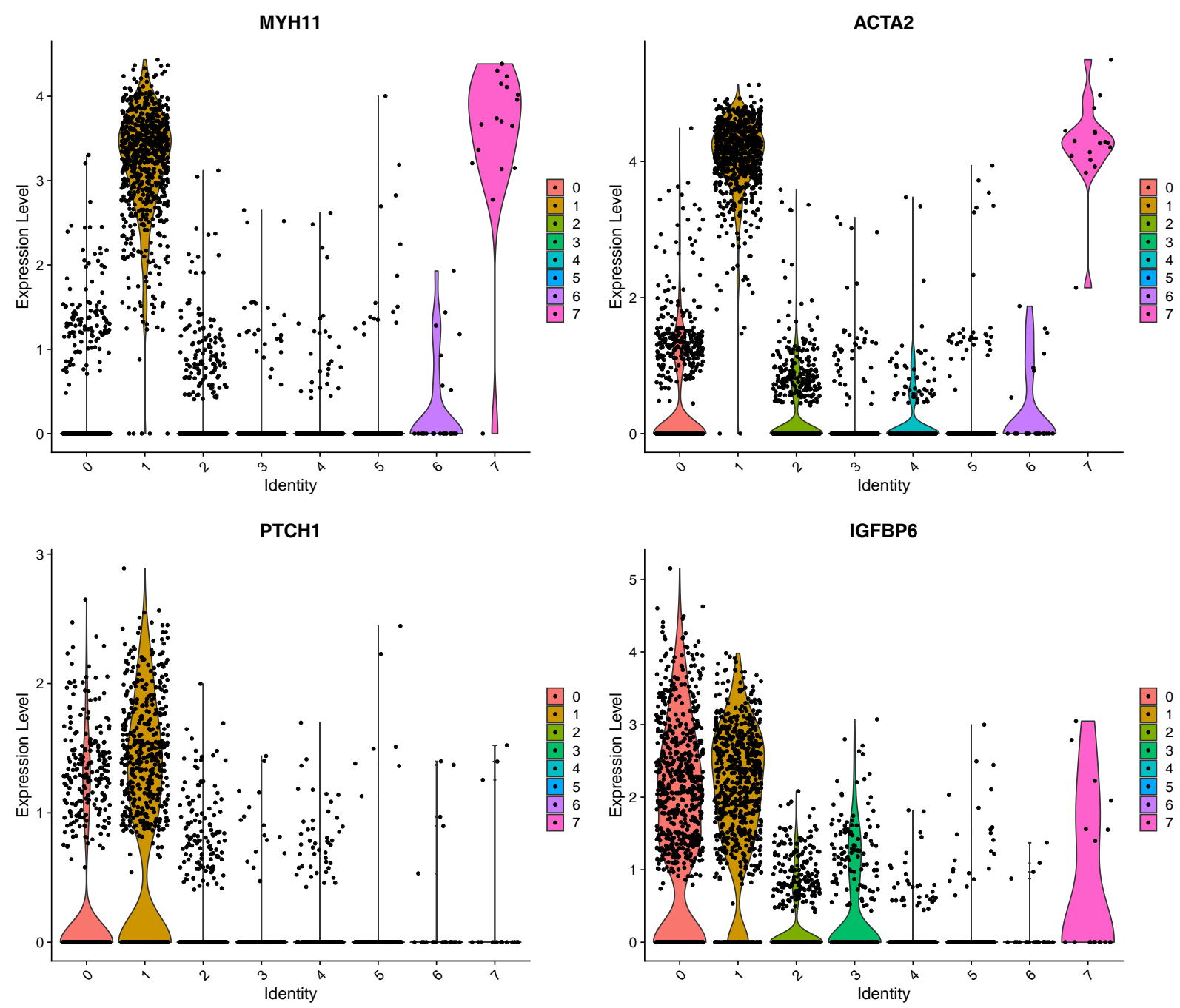

Figure S5 Gene expression patterns (violin plot) of selected markers in myoid cells. 

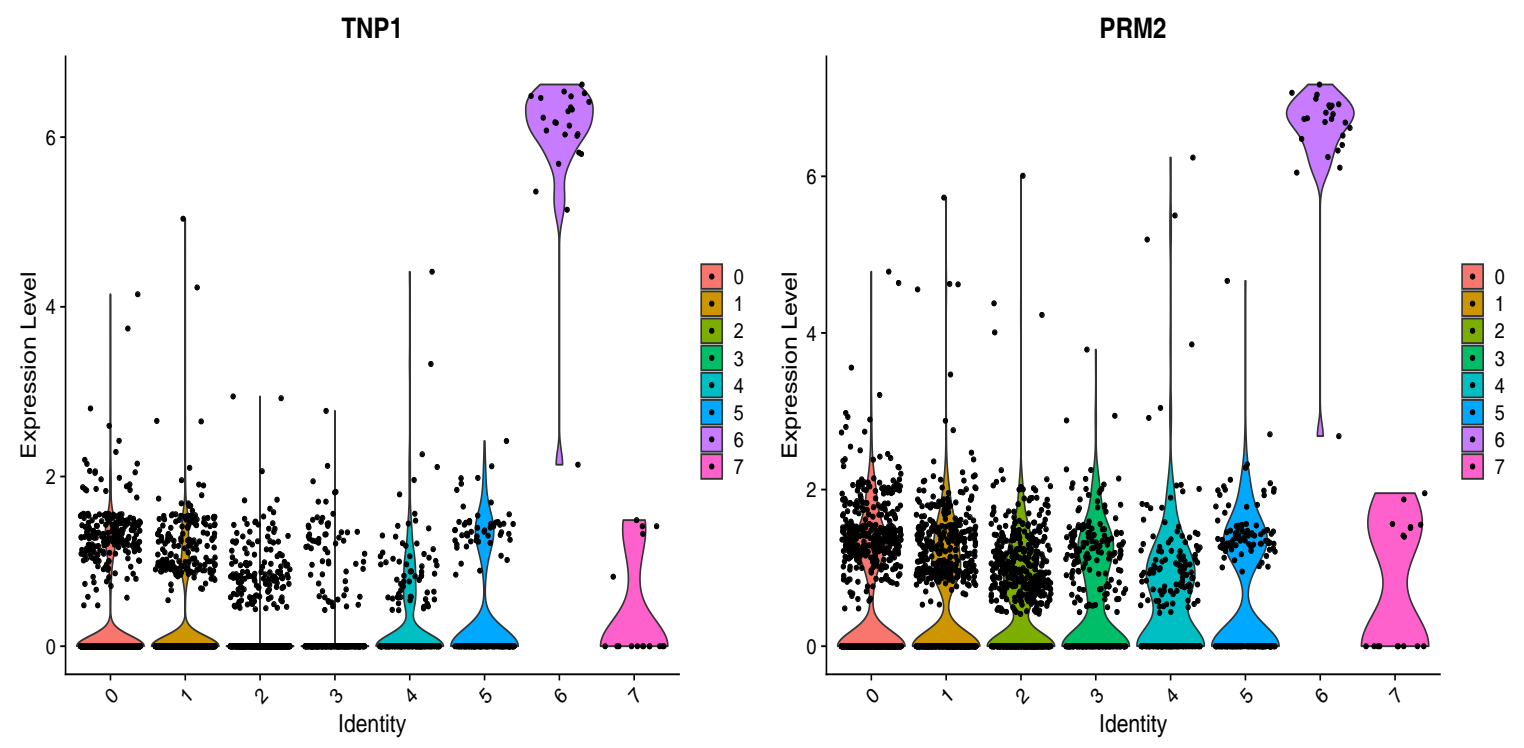

Figure S6 Gene expression patterns (violin plot) of selected markers in sperm.
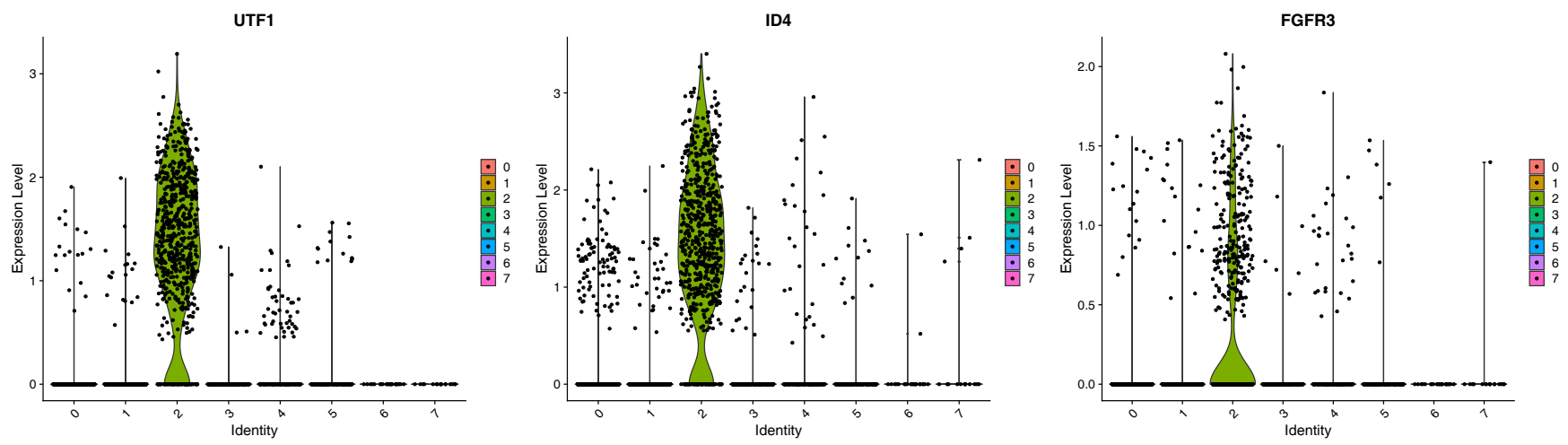

Figure S7 Gene expression patterns (violin plot) of selected markers in spermatogonial stem cells. 

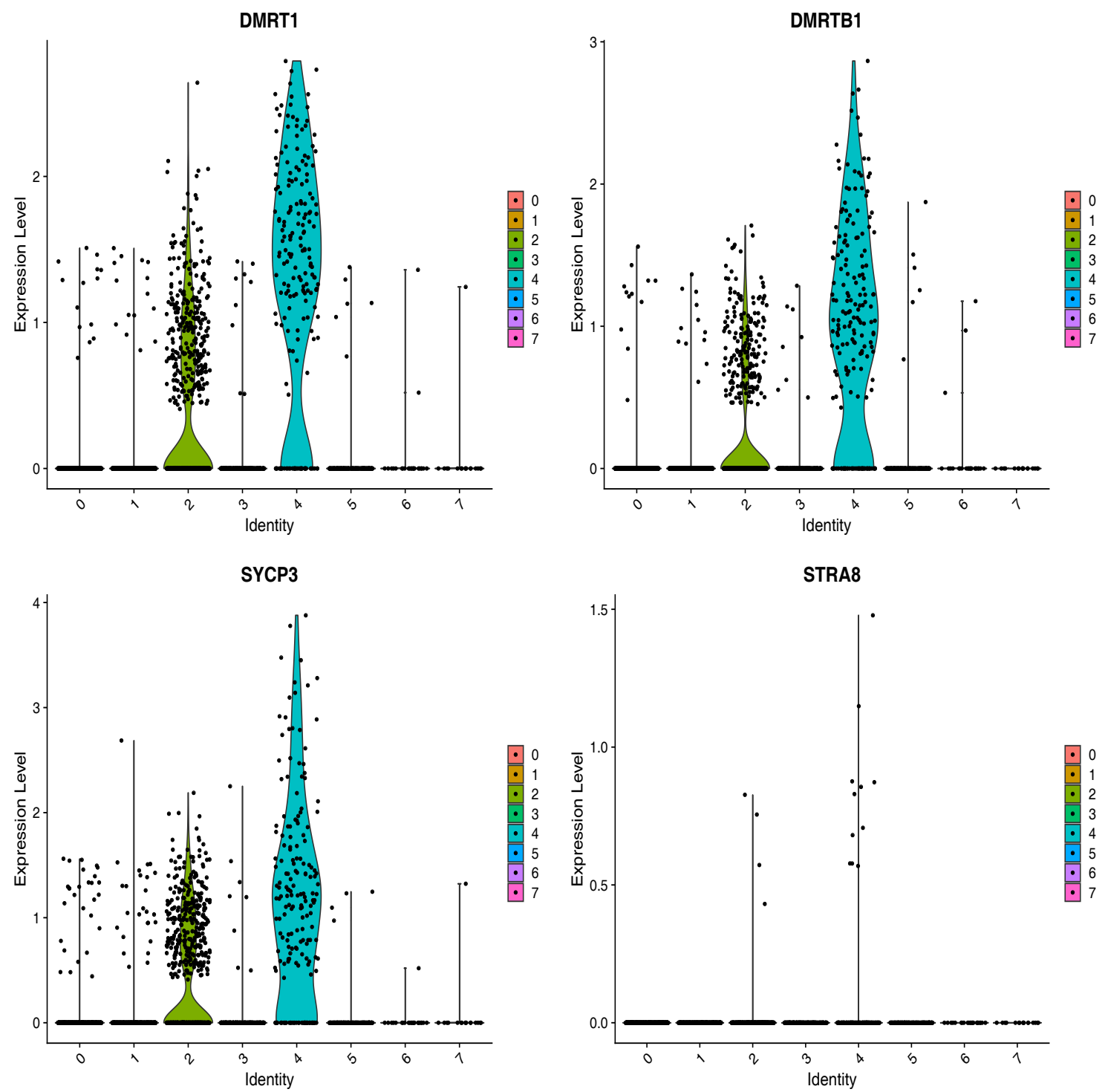

Figure S8 Gene expression patterns (violin plot) of selected markers in primary spermatocytes. 